\title{
Comparison of the Efficacy and Safety of Bexarotene and Photo(Chemo)Therapy Combination Therapy and Bexarotene Monotherapy for Cutaneous T-Cell Lymphoma
}

\author{
Akimichi Morita (D) C Chiharu Tateishi · Kyoko Ikumi · \\ Daisuke Hayashi · Aya Nakada · Haruna Nishihara · Kan Torii · \\ Emi Nishida $\cdot$ Daisuke Tsuruta
}

Received: October 9, 2021 / Accepted: November 25, 2021 / Published online: January 27, 2022

(C) The Author(s) 2022

\begin{abstract}
Introduction: Cutaneous T-cell lymphoma (CTCL) is a chronic condition with low malignancy. The combined use of therapeutic agents and photo(chemo)therapy is widely applied for the treatment of CTCL. The efficacy and safety of bexarotene and photo(chemo)therapy combination therapy were previously confirmed in Japanese patients with CTCL. The efficacy and safety of the bexarotene and photo(chemo)therapy combination therapy was compared with bexarotene monotherapy in Japanese patients with CTCL.
\end{abstract}

Supplementary Information The online version contains supplementary material available at https:// doi.org/10.1007/s13555-021-00655-0.

A. Morita $(\bowtie) \cdot$ K. Ikumi · A. Nakada .

H. Nishihara $\cdot$ K. Torii

Department of Geriatric and Environmental

Dermatology, Nagoya City University Graduate

School of Medical Sciences, 1 Kawasumi, Mizuhocho, Mizuho-ku, Nagoya 467-8601, Japan

e-mail: amorita@med.nagoya-cu.ac.jp

C. Tateishi · D. Hayashi · D. Tsuruta

Department of Dermatology, Osaka City University

Graduate School of Medicine, 1-4-3 Asahimachi,

Abeno-ku, Osaka 545-8585, Japan

\section{E. Nishida}

Department of Dermatology, Okazaki City Hospital, 3-1 Goshoai, Kouryuji-cho, Okazaki City, Aichi 4448553, Japan
Methods: This was a randomized, open-label, two-parallel-group, active-control specified clinical study in Japanese patients diagnosed with CTCL carried out over 8 weeks with a study extension conducted at two institutions. This study was registered in Japan Registry of Clinical Trials (jRCTs041180094).

Results: In the combination therapy group, 22 subjects received oral bexarotene $\left(300 \mathrm{mg} / \mathrm{m}^{2}\right.$ body surface area) once daily, followed by bathpsoralen and ultraviolet (UV) A or narrowband UVB. In the monotherapy group, 24 subjects received oral bexarotene $\left(300 \mathrm{mg} / \mathrm{m}^{2}\right)$ once daily. The efficacy analysis using the modified Severity-Weighted Assessment Tool, which included 39 patients, showed a response rate of $81.0 \%(17 / 21)$ in the combination therapy group and $83.3 \%(15 / 18)$ in the monotherapy group. No statistically significant difference was detected between groups. In the combination therapy group, four subjects showed a complete clinical response or complete response, and subjects with a partial response exhibited a high rate of skin lesion resolution, significantly better than in the monotherapy group. In the safety analysis, which included 46 treated subjects (22 in the combination therapy group and 24 in the monotherapy group), no adverse events or adverse drug reactions were reported in either group.

Conclusion: Both bexarotene and photo(chemo)therapy combination therapy and bexarotene monotherapy were 
therapeutically effective in Japanese patients with CTCL and well tolerated. Combination therapy led to a higher skin lesion resolution rate and greater therapeutic effects compared with monotherapy.

Trial Registration: jRCTs041180094.

\section{PLAIN LANGUAGE SUMMARY}

This study evaluated the efficacy and safety of bexarotene monotherapy compared with bexarotene and photo(chemo)therapy combination therapy in Japanese patients with cutaneous T-cell lymphoma (CTCL). The study was a randomized, open-label, two-parallelgroup, active-control specified clinical study in patients diagnosed with CTCL performed over an 8-week period with a study extension conducted in two institutions. In the combination therapy group, bexarotene $\left(300 \mathrm{mg} / \mathrm{m}^{2}\right.$ body surface area) was administered orally once daily to 22 subjects, followed by treatment with bathpsoralen and ultraviolet A (bath-PUVA) or narrowband UVB. In the bexarotene monotherapy group, bexarotene $\left(300 \mathrm{mg} / \mathrm{m}^{2}\right)$ was administered orally once daily to 24 subjects. Efficacy was assessed using the modified SeverityWeighted Assessment Tool. Among the 39 subjects analyzed for treatment efficacy, the response rate of the combination therapy group was $81.0 \%(17 / 21)$ and that of the monotherapy group was $83.3 \%(15 / 18)$. Differences between the two treatment groups were not statistically significant. Of the 21 subjects in the combination therapy group, 4 had a complete clinical response or complete response, and those with a partial response showed a higher skin lesion resolution rate than in the monotherapy group. The safety analysis revealed no reports of adverse events or adverse drug reactions among the 46 treated subjects (combination therapy group $=22$; monotherapy group $=24$ ). Thus, both bexarotene and photo(chemo)therapy combination therapy and bexarotene monotherapy were therapeutically effective and well tolerated in Japanese patients with CTCL. Patients receiving the combined therapy, however, showed a higher rate of skin lesion resolution.

Keywords: Bexarotene; Cutaneous T-cell lymphoma; Japanese; Phototherapy; Photochemotherapy

\section{Key Summary Points}

Why carry out this study?

Cutaneous T-cell lymphoma (CTCL) is generally treated with a combination of therapeutic agents and photo(chemo)therapy.

No studies to date have objectively compared the safety and efficacy of bexarotene monotherapy and combined bexarotene and photo(chemo)therapy in Japanese patients with CTCL.

What was learned from the study?

Bexarotene and photo(chemo)therapy combination therapy and bexarotene monotherapy are therapeutically effective in Japanese patients with CTCL and are well tolerated with no known adverse events or adverse drug reactions.

Bexarotene and photo(chemo)therapy combination therapy may induce a higher skin lesion resolution rate in Japanese patients with CTCL.

\section{INTRODUCTION}

Cutaneous T-cell lymphoma (CTCL) refers to a group of extranodal non-Hodgkin's lymphomas characterized by the infiltration of malignant T-cells into the skin [1-3]. Mycosis fungoides (MF), which accounts for most CTCL, occurs mainly in middle-aged and older people with a male-to-female ratio of 9:5 [4, 5]. Most patients have an indolent clinical course lasting > 10 years, and some cases progress over years 
from the patch to the plaque stage and eventually to the tumor stage. Patients whose disease state has progressed to the tumor stage develop organ infiltration and infections, leading to an extremely poor prognosis. During the disease course, the patient's quality of life markedly decreases because of the frequent occurrence or recurrence of cutaneous symptoms, which affect the patient's social life.

In recent years, international standardization of the diagnostic criteria and staging of CTCL has progressed, and treatment guidelines have been presented and disseminated in major regions of Europe and the USA by the World Health Organization and the European Organization for Research and Treatment of Cancer $[6,7]$. In 2018, Ibbotson provided an invaluable perspective on the use of psoralen and ultraviolet A (UVA) therapy (PUVA) and narrowband UVB for the management of a range of skin diseases, including CTCL [8]. The Japanese clinical practice guidelines indicate treatment modalities for each stage of CTCL. Phototherapy is used as a local treatment for patients with early- (stage IA-IIA) and advanced-stage (stage IIB and worse) CTCL [9]. Of the available photo(chemo)therapy methods, PUVA and narrowband UVB therapy are mainly used. A retrospective analysis of 62 patients with CTCL treated with PUVA in Japan demonstrated the efficacy of PUVA therapy. Patients who exhibit treatment resistance, however, might have a poor prognosis [10].

Clinical trials for developing new medical entities are complicated in Japan, and thus approval for treatments that are already used in Europe and the US is often delayed. In January 2016, bexarotene, one of the most popular CTCL treatments in Europe and the US, was approved for manufacture and sale in Japan for the management of CTCL.

The reports of clinical trial on the bexarotene are based mainly on the data of patients in Europe. Therefore, because of racial differences in photosensitivity due to variations in skin color, it is essential to conduct a clinical study on the use of combination therapy in Japanese patients. The evidence supporting the efficacy and safety of combined bexarotene and photo(chemo)therapy was provided recently
[11]. In that study, patients received daily oral dose of bexarotene calculated according to the body surface area $\left(300 \mathrm{mg} / \mathrm{m}^{2}\right)$, followed by bath-PUVA or narrowband UVB. At 24 weeks after initiating treatment, the total response rate was $80.0 \%$ according to the modified Severity-Weighted Assessment Tool (mSWAT) assessment [12] and 84.0\% using the Physician's Global Assessment (PGA). Response rates did not differ when stratified by disease stage. Comparison of bexarotene monotherapy with bexarotene and photo(chemo)therapy combination therapy, however, has not been reported in Japanese patients. Therefore, in the present study, the efficacy and safety were compared between bexarotene and photo(chemo)therapy combination therapy and bexarotene monotherapy in Japanese patients with CTCL.

\section{METHODS}

\section{Study Method}

This was a randomized, open-label, two-parallel-group, active-controlled specified clinical study in Japanese patients diagnosed with CTCL. This study was conducted at two sites (Nagoya City University Hospital and Osaka City University Hospital) under protocols reviewed and approved by Nagoya City University Hospital Clinical Research Review Board. In the case of multicenter specified clinical study under the Clinical Trials Act, after approval by the central Certified Review Board (CRB), the implementation permission of the administrator of each implementing medical institution has been obtained. The study conformed to the ethical principles of the Declaration of Helsinki (revised 2013) and the Clinical Trials Act in Japan. The outline of this study was registered and published in the Japan Registry of Clinical Trials (jRCT) (Trial ID; jRCTs041180094).

The major inclusion criteria were Japanese CTCL patients at least 20 years of age who provided written informed consent. The major exclusion criteria were as follows: contraindications to bexarotene use, pregnancy, breastfeeding, desire to become pregnant during the 
study period, having received topical CTCL treatment within 2 weeks prior to commencing treatment, treated with UVA or UVB irradiation within 3 weeks prior to commencing treatment, treated with radiation within 4 weeks before commencing treatment, and previous treatment with bexarotene.

In the bexarotene monotherapy group, bexarotene $\left(300 \mathrm{mg} / \mathrm{m}^{2}\right)$ was administered orally once daily after meals. Eight weeks after starting treatment, the treatment was terminated in subjects showing a partial response (PR) or better. In cases with stable disease (SD) or progressive disease (PD), photo(chemo)therapy was added, and combination treatment was performed for an additional 4 weeks. In the bexarotene and photo(chemo)therapy combination therapy group, the treatment was carried out as described previously [11]. Bexarotene $(300 \mathrm{mg} /$ $\mathrm{m}^{2}$ ) was administered orally once a day after meals.

\section{Irradiation Protocol for Bath-PUVA and Narrowband UVB}

Bath-PUVA or narrowband UVB was performed within $4 \mathrm{~h}$ after oral administration of bexarotene. For bath-PUVA, irradiation was started with $0.5 \mathrm{~J} / \mathrm{cm}^{2}$ UVA and performed five times weekly for 4 weeks after initiating the bexarotene therapy. The irradiation dose was increased by $0.5 \mathrm{~J} / \mathrm{cm}^{2}$ at each irradiation session (maximum, $4.0 \mathrm{~J} / \mathrm{cm}^{2}$ ). From 4 weeks after the initiation of bexarotene therapy, if the principal investigator or co-investigator judged that there were no issues related to subject's safety and if the patient's condition appeared to have improved, the irradiation dose or the number of irradiations was changed. For narrowband UVB, treatment was started at 50-70\% of the minimum erythema dose or $0.5-0.7 \mathrm{~J} /$ $\mathrm{cm}^{2}$ within $4 \mathrm{~h}$ after oral administration of bexarotene. Irradiation was performed five times weekly for 2 weeks after the initiation of bexarotene therapy, and the irradiation dose was increased by $20 \%$ at each irradiation session (maximum, $2.0 \mathrm{~J} / \mathrm{cm}^{2}$ ). From 2 weeks after the initiation of bexarotene therapy, when the principal investigator or co-investigator judged that there were no issues related to subject's safety and if the patient's condition appeared to have improved, the irradiation dose or the number of irradiations was changed.

\section{Efficacy Assessment}

Evaluation of the efficacy and safety was performed as follows. The following items were measured or surveyed on the first day of therapy at weeks $1,2,3,4,8,12$, and 24 or the day treatment was discontinued: mSWAT, PGA, safety, blood tests, blood biochemistry, body temperature, blood pressure, pulse rate, concomitant medication and therapy, subjective symptoms, objective findings, and drug compliance status. The staging and classification of MF and Sézary syndrome were conducted as described previously [13].

The mSWAT score was interpreted as follows: clinical complete response (CCR) or complete response (CR), $100 \%$ improvement in the mSWAT score from baseline; PR 50-99\% improvement; SD $25-50 \%$ improvement or $0-25 \%$ deterioration; and PD, deterioration of at least $25 \%$ or $>50 \%$ increase in the sum of the products of the greatest diameters of pathologically confirmed affected lymph nodes.

The PGA score was interpreted as follows: CCR, full resolution, no existence of the disease; $\mathrm{PR}, 50 \%$ or more improvement from baseline scores, existence of some trace of the disease or evidence of a certain degree of disease; $0-50 \%$ improvement or $25 \%$ deterioration or worse, significant remaining disease, or not significantly different from baseline; PD, 25\% deterioration or worse.

Evaluations at the commencement of treatment and after 4 weeks and 8 weeks were mandatory, while evaluations at 1,2 , and 3 weeks were optional. The final evaluation was performed after 12 weeks or at the time of study termination. For the safety evaluation, information on adverse events (AEs) was collected throughout the study period. 


\section{Statistical Analysis}

Statistical analysis was performed using SAS version 9.4 (SAS Institute, Cary, NC, USA), Microsoft Office Excel 2013, and Word 2013 (Microsoft, Redmond, WA, USA). The summary statistic of the weighted data (continuous amount) was set to the number of subjects, mean value, standard deviation, minimum value, median, and maximum value unless otherwise noted. For interval data, the number of target subjects and ratios were calculated.

\section{RESULTS}

\section{Patient Profiles}

The research design and study flow chart are shown in Fig. 1. A total of 53 subjects were enrolled in 2 institutions; 7 subjects withdrew from the study, and 46 subjects received the study drug. Dynamic allocation was performed by the minimization method using the adjustment factor (facility, disease type, and disease stage); the subjects were assigned to two groups at a ratio of 1:1 (bexarotene and photo[chemo]therapy combination therapy group $n=22$, and bexarotene monotherapy group $n=24$ ). All 46 subjects were included in the safety analysis set (SAS), but 1 subject in the combination therapy group and 5 subjects in the monotherapy group were excluded from the full analysis set (FAS) because the efficacy evaluation could not be performed because of treatment discontinuation. In addition, three subjects in the combination therapy group and five subjects in the monotherapy group were excluded from the per protocol set (PPS) for various reasons such as receiving a low dose of bexarotene (Fig. 1). The FAS was used for the efficacy analysis in this study.

\section{Efficacy}

Table 1 shows the background information of the subjects included in the SAS at the commencement of treatment. Mean age (mean [SD])

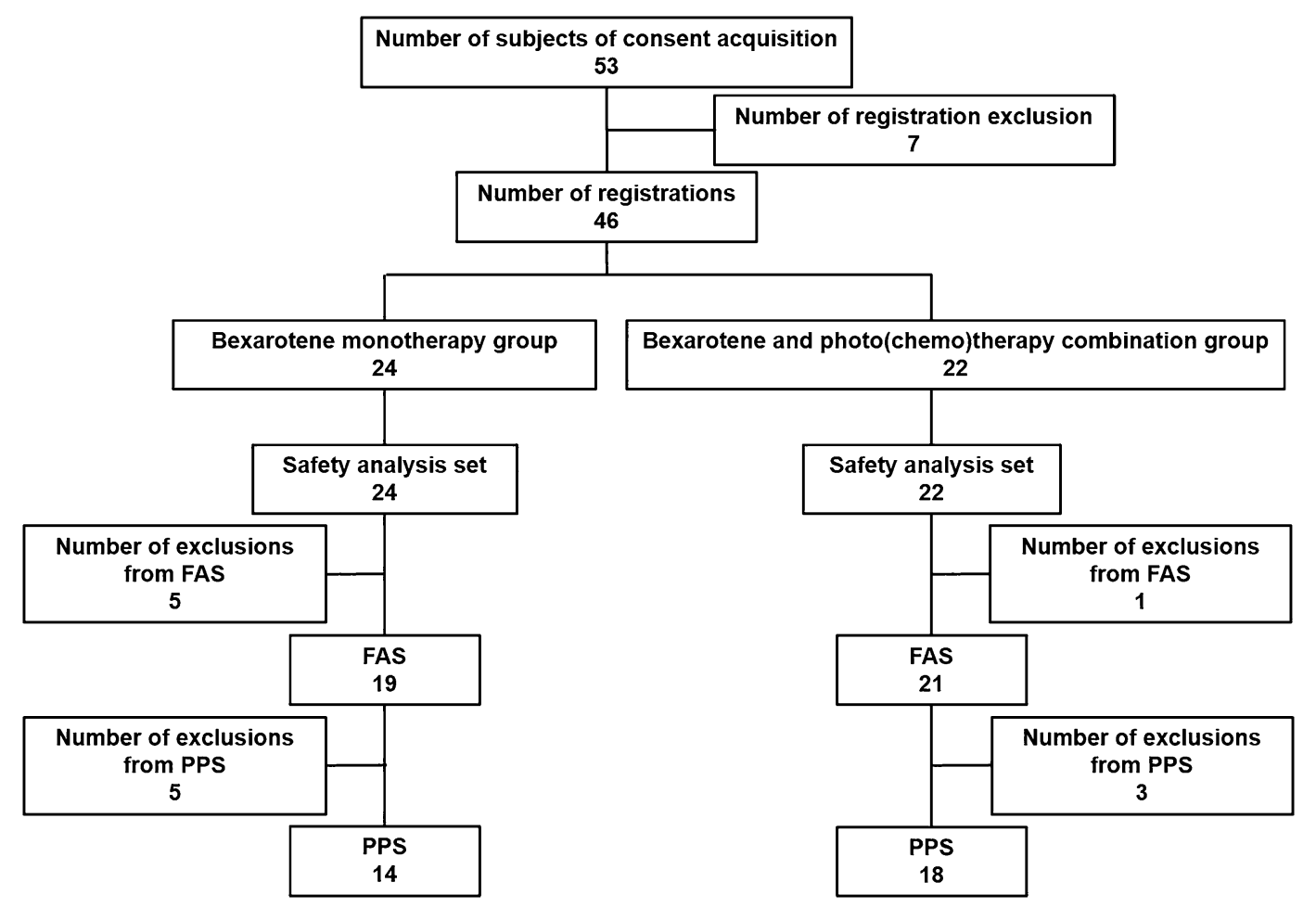

Fig. 1 Study design and subject flow chart. FAS full analysis set, PPS per protocol set 
Table 1 Baseline characteristics of patients with CTCL

\begin{tabular}{lllll}
\hline Category & Summary Statistics & Whole & Combination therapy & Monotherapy \\
\hline Sex & Male/female & $29(63.0) / 17(37.0)$ & $14(63.6) / 8(36.4)$ & $15(62.5) / 9(37.5)$ \\
Age category (years) & Mean \pm SD & $69.3 \pm 12.2$ & $68.3 \pm 13.3$ & $70.3 \pm 11.3$ \\
& Range & $31-91$ & $31-91$ & $42-84$ \\
& $<50$ & $4(8.7)$ & $2(9.1)$ & $2(8.3)$ \\
& $\geq 50$ to $<60$ & $5(10.9)$ & $3(13.6)$ & $2(8.3)$ \\
& $\geq 60$ to $<70$ & $8(17.4)$ & $3(13.6)$ & $5(20.8)$ \\
& $\geq 70$ to $<80$ & $20(43.5)$ & $11(50.0)$ & $9(37.5)$ \\
& $\geq 80$ & $9(19.6)$ & $3(13.6)$ & $6(25.0)$ \\
During of CTCL (years) & Mean \pm SD & $2.1 \pm 5.7$ & $1.9 \pm 5.2$ & $2.2 \pm 6.3$ \\
& Range & $0.0-28.7$ & $0.0-21.4$ & $0.0-28.7$ \\
Type of CTCL & Mycosis fungoides & $40(87.0)$ & $19(86.4)$ & $21(87.5)$ \\
& Sézary syndrome & $0(0.0)$ & $0(0.0)$ & $0(0.0)$ \\
& Others & $6(13.0)$ & $3(13.6)$ & $3(12.5)$ \\
Phase & $\leq$ IIA & $42(91.3)$ & $21(95.5)$ & $21(87.5)$ \\
BSA (m $\left.{ }^{2}\right)$ & $\geq$ IIB & $4(8.7)$ & $1(4.5)$ & $3(12.5)$ \\
& Mean \pm SD & $1.6 \pm 0.2$ & $1.7 \pm 0.2$ & $1.6 \pm 0.1$ \\
\hline Range & $1.3-2.1$ & $1.3-2.1$ & $1.3-1.8$ \\
\hline
\end{tabular}

Data are expressed as number (\%)

CTCL cutaneous T-cell lymphoma, $S D$ standard deviation, $B S A$ body surface area

in the combination therapy group was 68.3 [13.3] years (14 men [63.6\%] and 8 women [36.4\%]). Mean age (mean [SD]) in the monotherapy group was 70.3 [11.3] years (15 men [62.5\%] and 9 women [37.5\%]). Mean disease duration was $1.9 \pm 5.2$ years (combination therapy group) and $2.2 \pm 6.3$ years (monotherapy group). The CTCL disease type was MF in 19 subjects (86.4\%) in the combination therapy group and 21 subjects $(87.5 \%)$ in the monotherapy group; 3 subjects in each group had a different type of CTCL. The MF stage at the time of definite diagnosis was stage IIA or lower in 21 subjects $(95.5 \%)$ in the combination therapy group and in $21(87.5 \%)$ in the monotherapy group. One subject $(4.5 \%)$ in the combination therapy group and three subjects $(12.5 \%)$ in the monotherapy group had stage IIB or higher. Mean body surface area (mean
[SD]) was $1.7[0.2] \mathrm{m}^{2}$ in the combination therapy group and $1.6[0.1] \mathrm{m}^{2}$ in the monotherapy group. The main comorbidities were cataracts, hypertension, and dyslipidemia in the combination therapy group and hypertension, cataract, and hyperuricemia in the monotherapy group.

In the combination therapy group, 21 subjects received bath-PUVA as the photo(chemo)therapy with an integrated irradiation dose of $75.3[34.7] \mathrm{J} / \mathrm{cm}^{2}$ and 7 received narrowband UVB at an integrated dose of 11.2 [8.3] $\mathrm{J} / \mathrm{cm}^{2}$.

The results of the general skin lesion evaluation using the mSWAT at 8 weeks after the initiation of the study and the response rate of PR or better (CCR + CR + PR) are summarized in Table 2 (Supplementary Fig. 1). Regarding the response rate of PR or better, no difference was 
Table 2 Results of the mSWAT general skin lesion evaluation and the comprehensive evaluation of PGA

\begin{tabular}{|c|c|c|c|c|c|c|}
\hline \multirow{2}{*}{$\frac{\text { Group }}{\text { Whole }}$} & \multicolumn{3}{|c|}{ mSWAT } & \multicolumn{3}{|l|}{ PGA } \\
\hline & CCR & $1(2.6)$ & $\mathrm{CCR}+\mathrm{CR}+\mathrm{PR}$ & CCR & $4(10.3)$ & $\mathrm{CCR}+\mathrm{PR}$ \\
\hline & $\mathrm{CR}$ & $3(7.7)$ & $32(82.1)$ & & & $33(84.6)$ \\
\hline & $\mathrm{PR}$ & $28(71.8)$ & & $\mathrm{PR}$ & $29(74.4)$ & \\
\hline & SD & $7(17.9)$ & $\mathrm{SD}+\mathrm{PD}$ & SD & $6(15.4)$ & $\mathrm{SD}+\mathrm{PD}$ \\
\hline & $\mathrm{PD}$ & $0(0.0)$ & $7(17.9)$ & PD & $0(0.0)$ & $6(15.4)$ \\
\hline \multirow{5}{*}{$\begin{array}{l}\text { Bexarotene and } \\
\text { photo(chemo)therapy } \\
\text { combination therapy group }\end{array}$} & CCR & $1(4.8)$ & $\mathrm{CCR}+\mathrm{CR}+\mathrm{PR}$ & CCR & $4(19.0)$ & $\mathrm{CCR}+\mathrm{PR}$ \\
\hline & $\mathrm{CR}$ & $3(14.3)$ & $17(81.0)$ & & & $19(90.5)$ \\
\hline & $\mathrm{PR}$ & $13(61.9)$ & & PR & $15(71.4)$ & \\
\hline & $\mathrm{SD}$ & $4(19.0)$ & $\mathrm{SD}+\mathrm{PD}$ & SD & $2(9.5)$ & $\mathrm{SD}+\mathrm{PD}$ \\
\hline & $\mathrm{PD}$ & $0(0.0)$ & $4(19.0)$ & $\mathrm{PD}$ & $0(0.0)$ & $2(9.5)$ \\
\hline \multirow{5}{*}{$\begin{array}{l}\text { Bexarotene monotherapy } \\
\text { group }\end{array}$} & CCR & $0(0.0)$ & $\mathrm{CCR}+\mathrm{CR}+\mathrm{PR}$ & CCR & $0(0.0)$ & $\mathrm{CCR}+\mathrm{PR}$ \\
\hline & $\mathrm{CR}$ & $0(0.0)$ & $15(83.3)$ & & & $14(77.8)$ \\
\hline & $\mathrm{PR}$ & $15(83.3)$ & & $\mathrm{PR}$ & $14(77.8)$ & \\
\hline & SD & $3(16.7)$ & $\mathrm{SD}+\mathrm{PD}$ & SD & $4(22.2)$ & $\mathrm{SD}+\mathrm{PD}$ \\
\hline & $\mathrm{PD}$ & $0(0.0)$ & $3(16.7)$ & PD & $0(0.0)$ & $4(22.2)$ \\
\hline
\end{tabular}

Data are expressed as number (\%)

$C C R$ clinical complete response, $C R$ complete response, $P R$ partial response, $S D$ stable disease, $P D$ progressive disease

observed between the combination therapy group and the monotherapy group in $81.0 \%$ $(95 \%$ CI $[58.1,94.6])$ and $83.3 \%$ (95\% CI [58.6, 96.4]), respectively. The response rates of CR or better (CCR + CR) in the combination therapy group and monotherapy group were 19.0\% (4 subjects) and $0.0 \%$, respectively. Although no significant difference was observed, the response rate was higher in the combination therapy group. The change in mSWAT score over time was not significantly different between groups, but at each evaluation period, both groups had a significantly lower score compared with that at the start of treatment $(p<0.001)$, indicating that the mSWAT score decreased over time (Fig. 2).

The best overall response up to 8 weeks using the mSWAT scores is illustrated in Fig. 3 using waterfall plots. The waterfall plots showed a higher rate of skin lesion resolution in patients with a PR in the combination group.
The results of the comprehensive evaluation by physicians using the PGA after 8 weeks of treatment were aggregated to the response rate of PR or better (CCR + PR) in Table 3 (Supplementary Fig. 2). At the time of evaluation after 8 weeks, the response rate of the overall evaluation using the PGA was 90.5\% (95\% CI [69.6, $98.8]$ ) in the combination therapy group and $77.8 \%$ (95\% CI $[52.4,93.6])$ in the monotherapy group. The difference between groups was not statistically significant.

Based on the mSWAT score, the time to response (TTR) and time to PD (TTP) were calculated. The TTR from the date of commencing treatment to the time when the PR was confirmed for the first time was calculated using survival time analysis. The subjects that did not reach a PR by the time of the final evaluation were excluded from the final evaluation. The median TTR was 21.0 days (95\% CI [18.0, 22.0]) for the combination therapy group and 


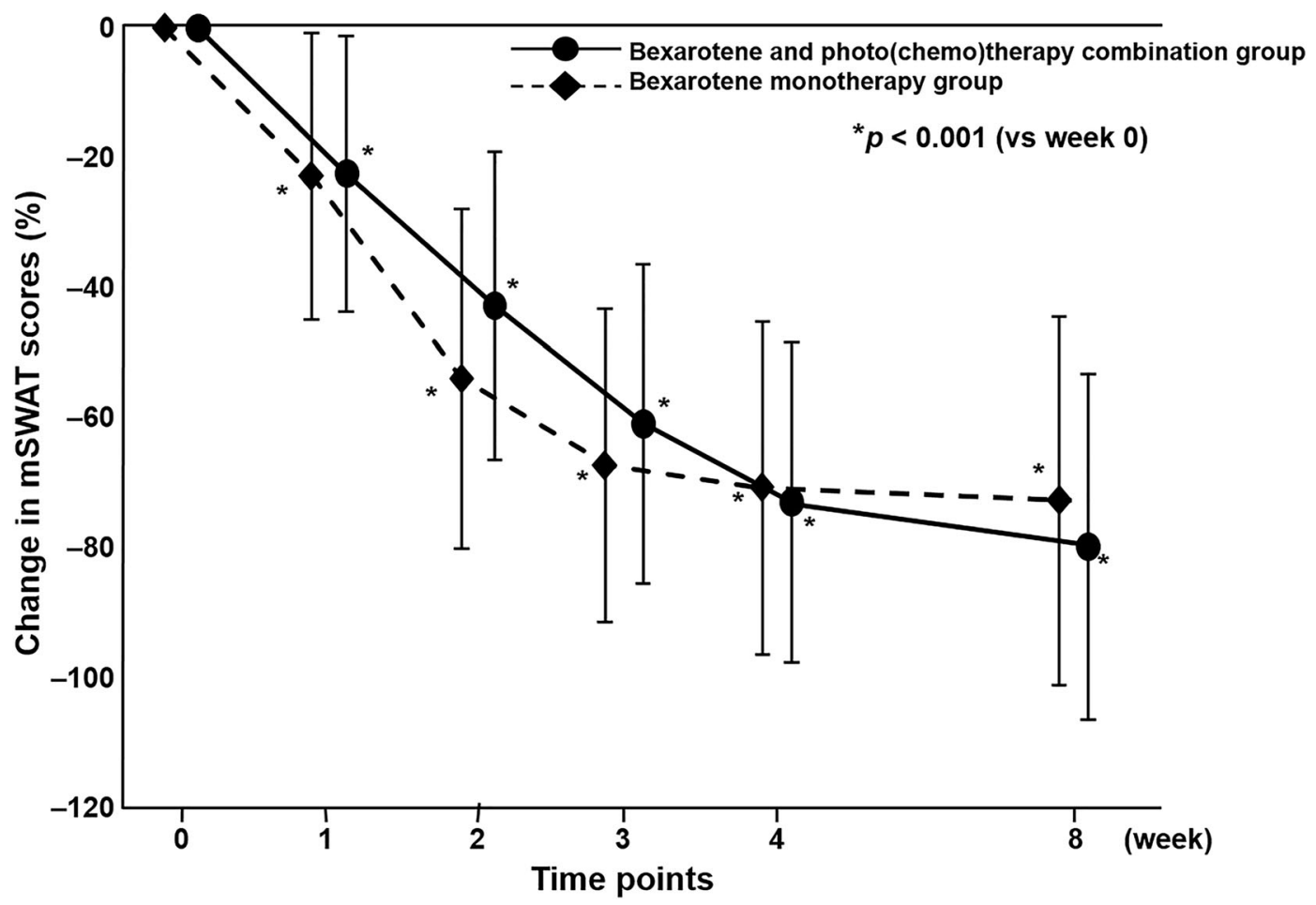

Fig. 2 Percentage change of overall cutaneous lesions based on the modified Severity-Weighted Assessment Tool (mSWAT) score. Circles with a solid line and rhombi with a dotted line indicate the bexarotene and

16.0 days (95\% CI $[14.0,21.0])$ for the monotherapy group. The difference between groups was not statistically significant (Fig. 4).

The TTP from the commencement of treatment to the time when it was determined to be advanced (PD) for the first time was calculated but could not calculate it because no cases showed disease progression.

\section{Safety}

All reported AEs were aggregated for the SAS. The major AEs reported are shown in Table 4. AEs were reported in all subjects, with a total of 231 events. The most severe AEs were hypertriglyceridemia, type 2 diabetes mellitus, acute cholecystitis, rhabdomyolysis, and interstitial lung disease.

A total of 164 adverse drug reactions (ADRs) occurred in all subjects (Table 4). The most serious ADRs reported were photo(chemo)therapy combination group and the bexarotene monotherapy group, respectively. Error bars represented standard deviation. ${ }^{*} p<0.001$ versus week 0

hypertriglyceridemia, rhabdomyolysis, and interstitial lung disease. The severity of other ADRs was mild to moderate. The ADRs with a high incidence were hypothyroidism, hypertriglyceridemia, hypercholesterolemia, and neutropenia. No new AEs or ADRs were reported that are not described in the package insert of the research drug.

\section{DISCUSSION}

The treatment strategy for malignant lymphoma is determined by the pathologic diagnosis, disease stage classification, and prognosis assessment [9]. The available therapeutic methods include skin-directed therapies, such as topical application, photo(chemo)therapy and radiation therapy, and systemic therapies, such as retinoid, interferon, and targeted therapies, alone or in combination. Skin-directed 
A

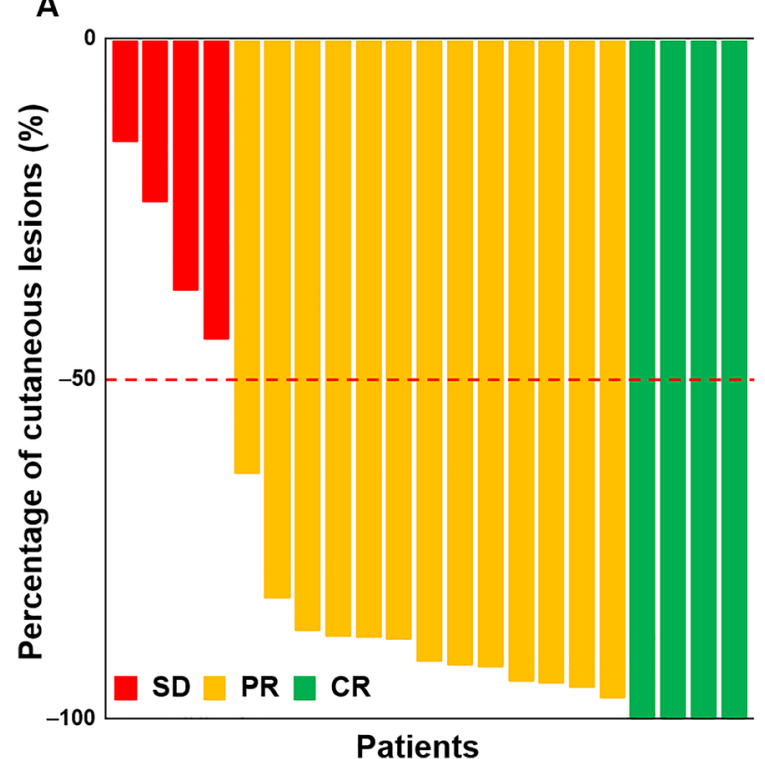

Fig. 3 Best overall responses in overall cutaneous lesions based on the mSWAT scores. A Bexarotene and photo(chemo)therapy combination group; $\mathbf{B}$ bexarotene

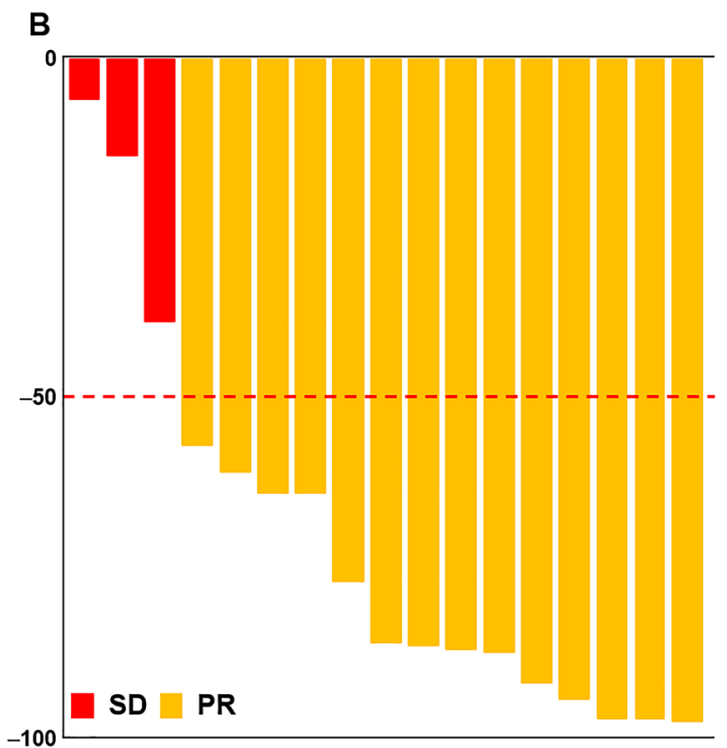

Patients

monotherapy group. $C R$ complete response, $P R$ partial response, $S D$ stable disease

A phase III clinical trial (NCT00056056; 93 subjects) published in 2012 as the first study comparing bexarotene and PUVA combination therapy with PUVA monotherapy for early CTCL found no significant difference in the response rates between the two groups [17]. Subjects in the bexarotene and PUVA combination therapy group who demonstrated a response, however, appeared to require less UV irradiation compared with subjects in the PUVA monotherapy group. The combination therapy was considered to reduce resistance to photo(chemo)therapy and suppress photo(chemo)therapy-induced ADRs by decreasing the required dose of UV irradiation.

In the present study, the efficacy and safety were compared between bexarotene and photo(chemo)therapy combination therapy and bexarotene monotherapy in Japanese patients with CTCL. Regarding the primary endpoints of this study, the response rates by mSWAT and PGA evaluation after 8 weeks were high in both groups (over $80 \%$ by mSWAT evaluation), and no significant difference was observed between the two groups. In both groups, the mSWAT scores decreased over the 
Table 3 Adverse events

\begin{tabular}{|c|c|c|c|c|c|c|}
\hline & \multicolumn{2}{|l|}{ Whole } & \multicolumn{2}{|c|}{$\begin{array}{l}\text { Combination } \\
\text { therapy }\end{array}$} & \multicolumn{2}{|c|}{ Monotherapy } \\
\hline & $\begin{array}{l}\text { Subjects } \\
(\%)\end{array}$ & $\overline{\text { Events }}$ & $\begin{array}{l}\text { Subjects } \\
(\%)\end{array}$ & $\overline{\text { Events }}$ & $\begin{array}{l}\text { Subjects } \\
(\%)\end{array}$ & $\overline{\text { Events }}$ \\
\hline Adverse events (overall) & $46(100.0)$ & 231 & $22(100.0)$ & 111 & $24(100.0)$ & 120 \\
\hline Metabolic and nutritional disorders & $44(95.7)$ & 83 & $21(95.5)$ & 39 & $23(95.8)$ & 44 \\
\hline Hypertriglyceridemia & $42(91.3)$ & 42 & $21(95.5)$ & 21 & $21(87.5)$ & 21 \\
\hline Hypercholesterolemia & $31(67.4)$ & 31 & $14(63.6)$ & 14 & $17(70.8)$ & 17 \\
\hline Hyperuricemia & $3(6.5)$ & 3 & $1(4.5)$ & 1 & $2(8.3)$ & 2 \\
\hline Hypoalbuminemia & $3(6.5)$ & 3 & $2(9.1)$ & 2 & $1(4.2)$ & 1 \\
\hline Type 2 diabetes & $1(2.2)$ & 1 & $0(0.0)$ & 0 & $1(4.2)$ & 1 \\
\hline Endocrine disorders & $43(93.5)$ & 43 & $22(100.0)$ & 22 & $21(87.5)$ & 21 \\
\hline Hypothyroidism & $43(93.5)$ & 43 & $22(100.0)$ & 22 & $21(87.5)$ & 21 \\
\hline Laboratory test & $24(52.2)$ & 27 & $10(45.5)$ & 10 & $14(58.3)$ & 17 \\
\hline CRP increase & $17(37.0)$ & 17 & $7(31.8)$ & 7 & $10(41.7)$ & 10 \\
\hline Increased platelet count & $6(6.5)$ & 3 & $2(9.1)$ & 2 & $1(4.2)$ & 1 \\
\hline Increased blood creatine phosphokinase & $6(6.5)$ & 3 & $0(0.0)$ & 0 & $3(12.5)$ & 3 \\
\hline Blood and lymphatic disorders & $19(41.3)$ & 23 & $9(40.9)$ & 11 & $10(41.7)$ & 12 \\
\hline Neutropenia & $16(34.8)$ & 16 & $7(31.8)$ & 7 & $9(37.5)$ & 9 \\
\hline Anemia & $5(10.9)$ & 5 & $3(13.6)$ & 3 & $2(8.3)$ & 2 \\
\hline Gastrointestinal disorders & $8(17.4)$ & 10 & $3(13.6)$ & 5 & $5(20.8)$ & 5 \\
\hline Coprostasis & $3(6.5)$ & 3 & $2(9.1)$ & 2 & $1(4.2)$ & 1 \\
\hline Hepatic biliary disorders & $7(15.2)$ & 7 & $2(9.1)$ & 2 & $5(20.8)$ & 5 \\
\hline Liver function abnormality & $6(13.0)$ & 6 & $2(9.1)$ & 2 & $4(16.7)$ & 4 \\
\hline Acute cholecystitis & $1(2.2)$ & 1 & $0(0.0)$ & 0 & $1(4.2)$ & 1 \\
\hline $\begin{array}{l}\text { General and systemic disorders and conditions at the site } \\
\text { of administration }\end{array}$ & $6(13.0)$ & 8 & $4(18.2)$ & 6 & $2(8.3)$ & 2 \\
\hline Fatigue & $3(6.5)$ & 4 & $2(9.1)$ & 3 & $1(4.2)$ & 1 \\
\hline Skin and subcutaneous tissue disorders & $4(8.7)$ & 6 & $3(13.6)$ & 5 & $1(4.2)$ & 1 \\
\hline Skin dryness & $2(4.3)$ & 2 & $2(9.1)$ & 2 & $0(0.0)$ & 0 \\
\hline Musculoskeletal and connective tissue disorders & $4(8.7)$ & 4 & $4(4.5)$ & 1 & $3(12.5)$ & 3 \\
\hline Rhabdomyolysis & $1(2.2)$ & 1 & $0(0.0)$ & 0 & $1(4.2)$ & 1 \\
\hline Nervous system disorders & $3(6.5)$ & 3 & $2(9.1)$ & 2 & $1(4.2)$ & 1 \\
\hline Headache & $3(6.5)$ & 3 & $2(9.1)$ & 2 & $1(4.2)$ & 1 \\
\hline Respiratory, thorax, and longitudinal disorders & $1(2.2)$ & 1 & $1(4.5)$ & 1 & $0(0.0)$ & 0 \\
\hline
\end{tabular}


Table 3 continued

\begin{tabular}{|c|c|c|c|c|c|c|}
\hline & \multicolumn{2}{|l|}{ Whole } & \multicolumn{2}{|c|}{$\begin{array}{l}\text { Combination } \\
\text { therapy }\end{array}$} & \multicolumn{2}{|c|}{ Monotherapy } \\
\hline & $\begin{array}{l}\text { Subjects } \\
(\%)\end{array}$ & Events & $\begin{array}{l}\text { Subjects } \\
(\%)\end{array}$ & Events & $\begin{array}{l}\text { Subjects } \\
(\%)\end{array}$ & Events \\
\hline Interstitial lung disease & $1(2.2)$ & 1 & $1(4.5)$ & 1 & $0(0.0)$ & 0 \\
\hline
\end{tabular}

Data are expressed as number (\%)

8-week study period, but four subjects in the bexarotene and photo(chemo)therapy combination therapy group had a CR or better $(\mathrm{CCR}+\mathrm{CR})$, leading to a lower final mean score in the combination therapy group. In our previous study, bexarotene and photo(chemo)therapy combination therapy led to a response rate (based on the mSWAT score) of $80 \%$ (20/25 patients) in the FAS and 75\% (9/ 12 cases) in the PPS; even when limiting the disease stage to IIA or higher, the response rate was $75 \%$ (9/12 patients) in the PPS [11]. The subjects in this study had a high proportion of early-stage MF and high response rates by mSWAT and PGA evaluation after 8 weeks, suggesting that early diagnosis and early treatment are necessary for CTCL treatment. A case series study performed by Fujimura et al. indicated that a low initial dose of bexarotene (150-300 mg/body) combined with narrowband UVB could be an optimal treatment for advanced-stage CTCL [18]. These findings

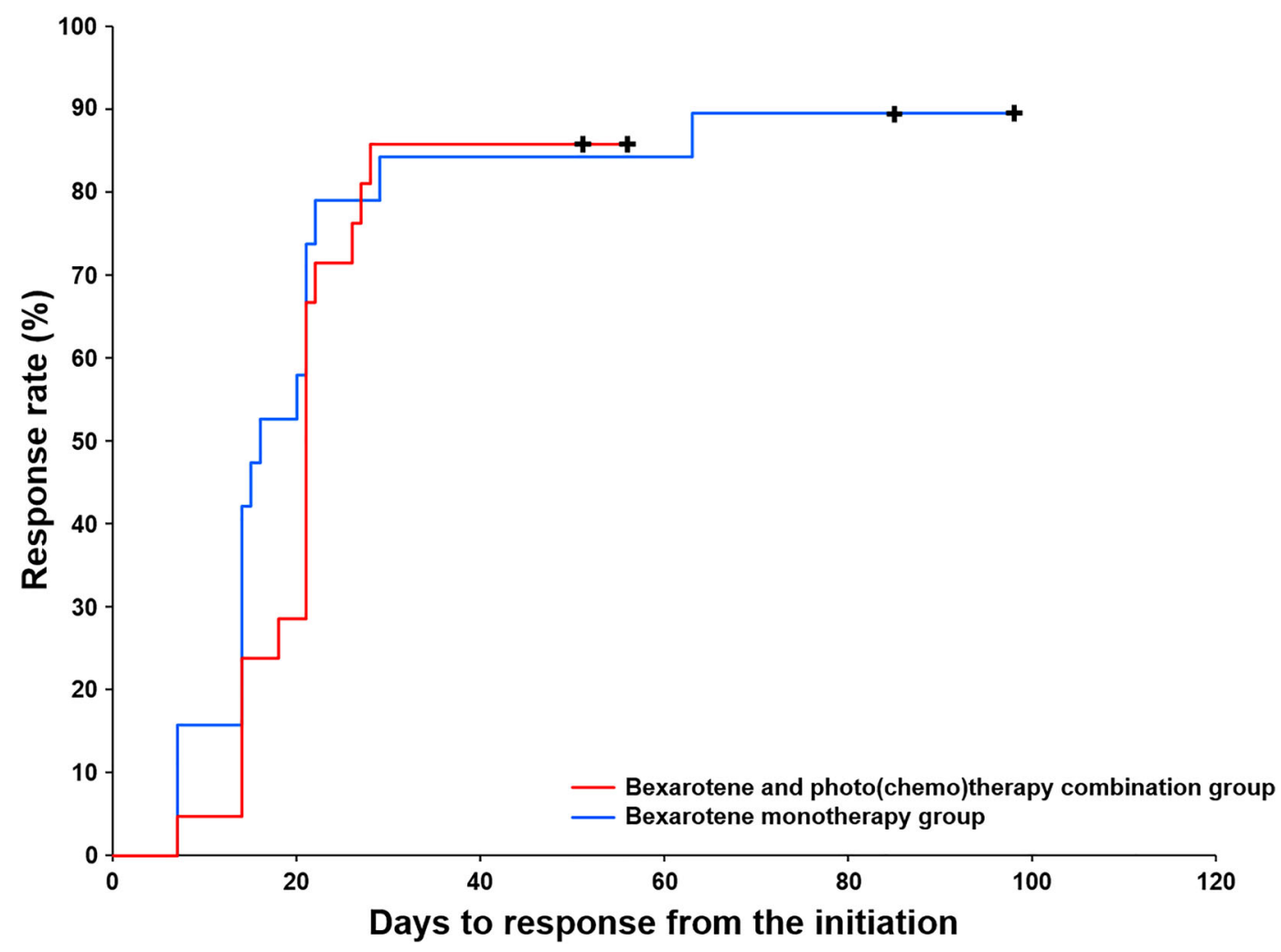

Fig. 4 Time to response (TTR) of the full analysis set. Red and blue lines indicate the bexarotene and photo(chemo)therapy combination group and the bexarotene monotherapy group, respectively 
Table 4 Adverse events

\begin{tabular}{|c|c|c|c|c|c|c|}
\hline & \multicolumn{2}{|l|}{ Whole } & \multicolumn{2}{|c|}{ Combination therapy } & \multicolumn{2}{|l|}{ Monotherapy } \\
\hline & Subjects (\%) & Events & Subjects (\%) & Events & Subjects (\%) & Events \\
\hline Adverse events (overall) & $46(100.0)$ & 231 & $22(100.0)$ & 111 & $24(100.0)$ & 120 \\
\hline Metabolic and nutritional disorders & $44(95.7)$ & 83 & $21(95.5)$ & 39 & $23(95.8)$ & 44 \\
\hline Hypertriglyceridemia & $42(91.3)$ & 42 & $21(95.5)$ & 21 & $21(87.5)$ & 21 \\
\hline Hypercholesterolemia & $31(67.4)$ & 31 & $14(63.6)$ & 14 & $17(70.8)$ & 17 \\
\hline Hyperuricemia & $3(6.5)$ & 3 & $1(4.5)$ & 1 & $2(8.3)$ & 2 \\
\hline Hypoalbuminemia & $3(6.5)$ & 3 & $2(9.1)$ & 2 & $1(4.2)$ & 1 \\
\hline Type 2 diabetes & $1(2.2)$ & 1 & $0(0.0)$ & 0 & $1(4.2)$ & 1 \\
\hline Endocrine disorders & $43(93.5)$ & 43 & $22(100.0)$ & 22 & $21(87.5)$ & 21 \\
\hline Hypothyroidism & $43(93.5)$ & 43 & $22(100.0)$ & 22 & $21(87.5)$ & 21 \\
\hline Laboratory test & $24(52.2)$ & 27 & $10(45.5)$ & 10 & $14(58.3)$ & 17 \\
\hline CRP increase & $17(37.0)$ & 17 & $7(31.8)$ & 7 & $10(41.7)$ & 10 \\
\hline Increased platelet count & $6(6.5)$ & 3 & $2(9.1)$ & 2 & $1(4.2)$ & 1 \\
\hline $\begin{array}{l}\text { Increased blood creatine } \\
\text { phosphokinase }\end{array}$ & $6(6.5)$ & 3 & $0(0.0)$ & 0 & $3(12.5)$ & 3 \\
\hline Blood and lymphatic disorders & $19(41.3)$ & 23 & $9(40.9)$ & 11 & $10(41.7)$ & 12 \\
\hline Neutropenia & $16(34.8)$ & 16 & $7(31.8)$ & 7 & $9(37.5)$ & 9 \\
\hline Anemia & $5(10.9)$ & 5 & $3(13.6)$ & 3 & $2(8.3)$ & 2 \\
\hline Gastrointestinal disorders & $8(17.4)$ & 10 & $3(13.6)$ & 5 & $5(20.8)$ & 5 \\
\hline Coprostasis & $3(6.5)$ & 3 & $2(9.1)$ & 2 & $1(4.2)$ & 1 \\
\hline Hepatic biliary disorders & $7(15.2)$ & 7 & $2(9.1)$ & 2 & $5(20.8)$ & 5 \\
\hline Liver function abnormality & $6(13.0)$ & 6 & $2(9.1)$ & 2 & $4(16.7)$ & 4 \\
\hline Acute cholecystitis & $1(2.2)$ & 1 & $0(0.0)$ & 0 & $1(4.2)$ & 1 \\
\hline $\begin{array}{l}\text { General and systemic disorders } \\
\text { and conditions at the site } \\
\text { of administration }\end{array}$ & $6(13.0)$ & 8 & $4(18.2)$ & 6 & $2(8.3)$ & 2 \\
\hline Fatigue & $3(6.5)$ & 4 & $2(9.1)$ & 3 & $1(4.2)$ & 1 \\
\hline $\begin{array}{l}\text { Skin and subcutaneous tissue } \\
\text { disorders }\end{array}$ & $4(8.7)$ & 6 & $3(13.6)$ & 5 & $1(4.2)$ & 1 \\
\hline Skin dryness & $2(4.3)$ & 2 & $2(9.1)$ & 2 & $0(0.0)$ & 0 \\
\hline
\end{tabular}


Table 4 continued

\begin{tabular}{|c|c|c|c|c|c|c|}
\hline & \multicolumn{2}{|l|}{ Whole } & \multicolumn{2}{|c|}{ Combination therapy } & \multicolumn{2}{|l|}{ Monotherapy } \\
\hline & $\overline{\text { Subjects (\%) }}$ & $\overline{\text { Events }}$ & Subjects (\%) & $\overline{\text { Events }}$ & Subjects (\%) & $\overline{\text { Events }}$ \\
\hline $\begin{array}{l}\text { Musculoskeletal and connective } \\
\text { tissue disorders }\end{array}$ & $4(8.7)$ & 4 & $4(4.5)$ & 1 & $3(12.5)$ & 3 \\
\hline Rhabdomyolysis & $1(2.2)$ & 1 & $0(0.0)$ & 0 & $1(4.2)$ & 1 \\
\hline Nervous system disorders & $3(6.5)$ & 3 & $2(9.1)$ & 2 & $1(4.2)$ & 1 \\
\hline Headache & $3(6.5)$ & 3 & $2(9.1)$ & 2 & $1(4.2)$ & 1 \\
\hline $\begin{array}{l}\text { Respiratory, thorax, and } \\
\text { longitudinal disorders }\end{array}$ & $1(2.2)$ & 1 & $1(4.5)$ & 1 & $0(0.0)$ & 0 \\
\hline Interstitial lung disease & $1(2.2)$ & 1 & $1(4.5)$ & 1 & $0(0.0)$ & 0 \\
\hline
\end{tabular}

Data are expressed as number (\%)

suggest the usefulness of bexarotene as adjuvant therapy for photo(chemo)therapy.

The results of the mSWAT and PGA evaluations after 8 weeks did not differ significantly between the two groups. Our study results are consistent with the findings of Whittaker et al. [17], who reported no significant difference in the response rate or response duration in a randomized clinical trial of bexarotene and PUVA combination therapy and bexarotene monotherapy in European early-stage MF patients; the best overall response rate was $71 \%$ for PUVA monotherapy and $77 \%$ for combination therapy. In the present study, the integrated irradiation dose in PUVA was $75.3 \pm 34.7 \mathrm{~J} / \mathrm{cm}^{2}$, comparable to that in Whittaker et al. $\left(101.7 \mathrm{~J} / \mathrm{cm}^{2}\right)$ [17].

The two groups did not differ in terms of the TTR and the TTP by mSWAT evaluation, which are secondary endpoints, because response rates in both groups reached $>80 \%$ within 30 days. The high response rate in the present study is likely attributable to: (1) the inclusion of many early-stage MF patients and (2) topical application of various steroids. These findings suggest the usefulness of bexarotene for earlier resolution of skin symptoms in early-stage MF patients.

Although ADRs of bexarotene are reported in Japan and elsewhere, none were specific to this study. There were three cases of discontinuation of the study due to ADRs. The AEs and ADRs that occurred were reported, with a total of 231 and 164 events, respectively. The AEs and ADRs that occurred frequently were hypothyroidism, hypertriglyceridemia, hypercholesterolemia, and neutropenia, all of which are known. Serious AEs and ADRs were observed in five events in five subjects, and in three events in three subjects, respectively, but all patients recovered after receiving medical treatment.

Bexarotene exhibits phototoxicity in in vitro tests (photo-hemolytic test and histidine photooxidation reaction) [19]. In previous studies, non-serious photosensitivity was reported among those receiving combination therapy with UVB irradiation: $6.3 \%(1 / 16)$ patients in a phase I/II study (B-1101 study) in Japan $[19,20]$, $1.7 \%(1 / 59)$ patients in a phase IV study (E7273G000-401 study) (2019, pers. comm.), and 1.7\% $(1 / 58)$ patients in phase II/III study (L1069-23 study) performed in the US [21]. No photosensitivity was reported in the present study.

\section{CONCLUSIONS}

This study revealed no statistical difference in the therapeutic effect between combined bexarotene and photo(chemo)therapy and bexarotene monotherapy based on the mSWAT and PGA evaluation after 8 weeks. Both the combination therapy and monotherapy, however, 
were efficacious for the management of CTCL. Moreover, in the bexarotene and photo(chemo)therapy combination group, some patients showed a complete response, suggesting that the combination of both bexarotene and photo(chemo)therapy has a greater therapeutic effect than bexarotene monotherapy in Japanese patients with CTCL.

\section{ACKNOWLEDGEMENTS}

The authors thank all the study participants for their involvement in the study.

Funding. This research and the journal's Rapid Service Fees were funded by Minophagen Pharmaceutical Co., Ltd., Tokyo, Japan.

Authorship. All named authors meet the International Committee of Medical Journal Editors criteria for authorship for this article, take responsibility for the integrity of the work as a whole, and have given their approval for this version to be published.

Author Contributions. All authors contributed to the study conception, design, material preparation and data collection. Data analysis was performed by Akimichi Morita. The first draft of the manuscript was written by Akimichi Morita and all authors commented on previous versions of the manuscript. All authors read and approved the final manuscript.

Disclosures. Akimichi Morita, Chiharu Tateishi, and Daisuke Tsuruta have received research grants from Minophagen Pharmaceutical Co., Ltd. and received a speaker's fee. Kyoko Ikumi, Daisuke Hayashi, Aya Nakada, Haruna Nishihara, Kan Torii and Emi Nishid have nothing to disclose.

Compliance with Ethical Guidelines. This study was conducted in accordance with protocols reviewed and approved by Nagoya City University Hospital Clinical Research Review board and conformed to the ethical principles of the Declaration of Helsinki (revised 2013) and the Clinical Trials Act in Japan. In the case of multicenter specified clinical study under the Clinical Trials Act, after approval by the central Certified Review Board (CRB), the implementation permission of the administrator of each implementing medical institution has been obtained. The outline of this study is registered and published in the Japan Registry of Clinical Trials (jRCT) (Trial ID; jRCTs041180094). The patients provided written informed consent for publication of any identifying information after receiving an explanation of the study.

Data Availability. The datasets generated and/or analyzed during the current study are available from the corresponding author on reasonable request.

Open Access. This article is licensed under a Creative Commons Attribution-NonCommercial 4.0 International License, which permits any non-commercial use, sharing, adaptation, distribution and reproduction in any medium or format, as long as you give appropriate credit to the original author(s) and the source, provide a link to the Creative Commons licence, and indicate if changes were made. The images or other third party material in this article are included in the article's Creative Commons licence, unless indicated otherwise in a credit line to the material. If material is not included in the article's Creative Commons licence and your intended use is not permitted by statutory regulation or exceeds the permitted use, you will need to obtain permission directly from the copyright holder. To view a copy of this licence, visit http://creativecommons.org/licenses/by$\mathrm{nc} / 4.0 /$.

\section{REFERENCES}

1. Dores GM, Anderson WF, Devesa SS. Cutaneous lymphomas reported to the national cancer institute's surveillance, epidemiology, and end results program: applying the new WHO-European organization for research and treatment of cancer classification system. J Clin Oncol. 2005;23:7246-8.

2. Willemze R, Jaffe ES, Burg G, et al. WHO-EORTC classification for cutaneous lymphomas. Blood. 2005;105:3768-85. 
3. Zackheim HS, Vonderheid EC, Ramsay DL, LeBoit PE, Rothfleisch J, Kashani-Sabet M. Relative frequency of various forms of primary cutaneous lymphomas. J Am Acad Dermatol. 2000;43:793-6.

4. Morton LM, Wang SS, Devesa SS, Hartge P, Weisenburger DD, Linet MS. Lymphoma incidence patterns by WHO subtype in the United States, 1992-2001. Blood. 2006;107:265-76.

5. Aoki R, Karube K, Sugita Y, et al. Distribution of malignant lymphoma in Japan: analysis of 2260 cases, 2001-2006. Pathol Int. 2008;58:174-82.

6. National Comprehensive Cancer Network. Primary cutaneous lymphomas (Version 4.2019). NCCN Guidelines, Plymouth Meeting, PA; [Cited 2020 Jan 12]. Available from: https://www.nccn.org/ professionals/physician_gls/pdf/primary_ cutaneous.pdf.

7. Trautinger F, Eder J, Assaf C, et al. European Organization for Research and Treatment of Cancer consensus recommendations for the treatment of mycosis fungoides/Sezary syndrome-update 2017. Eur J Cancer. 2017;77:57-74.

8. Ibbotson SH. A perspective on the use of NB-UVB phototherapy vs. PUVA photochemotherapy. Front Med. 2018;5:184-99.

9. Ohtsuka M, Hamada T, Miyagaki T, et al. Outlines of the Japanese guidelines for the management of primary cutaneous lymphomas 2020. J Dermatol. 2021;48:e49-71.

10. Shintani Y, Nishida E, Furuhashi T, et al. Efficacy of bath-psoralen and ultraviolet A therapy for mycosis fungoides-retrospective analysis of 62 cases. J Dermatol. 2021. https://doi.org/10.1111/1346-8138. 16077 (in press).

11. Morita A, Tateishi C, Muramatsu S, et al. Efficacy and safety of bexarotene combined with photo(chemo)therapy for cutaneous T-cell lymphoma. J Dermatol. 2020;47:443-51.

12. Olsen EA, Kim YH, Kuzel TM, et al. Phase IIb multicenter trial of vorinostat in patients with persistent, progressive, or treatment refractory cutaneous T-cell lymphoma. J Clin Oncol. 2007;25:3109-15.

13. Olsen EA, Whittaker S, Kim YH, et al. Clinical end points and response criteria in mycosis fungoides and Sézary syndrome: a consensus statement of the international society for cutaneous lymphomas, the United States cutaneous lymphoma consortium, and the cutaneous lymphoma task force of the European organization for research and treatment of cancer. J Clin Oncol. 2011;29:2598-607.

14. Duvic M, Martin AG, Kim Y, et al. Phase 2 and 3 clinical trials of oral bexarotene (Targretin capsule s) for the treatment of refractory or persistent earlystage cutaneous T-cell lymphoma. Arch Dermatol. 2001;137:581-93.

15. Ahmad K, Rogers S, McNicholas PD, Collins P. Narrowband UVB and PUVA in the treatment of mycosis fungoides: a retrospective study. Acta Derm Venereol. 2007;87:413-7.

16. Whittaker SJ, Marsden JR, Spittle M, Russell JR. Joint British Association of Dermatologists and U.K. Cutaneous Lymphoma Group guidelines for the management of primary cutaneous T-cell lymphomas. Br J Dermatol. 2003;149:1095-107.

17. Whittaker S, Oritz P, Dummer R, et al. Efficacy and safety of bexarotene combined with psoralen-ultraviolet A (PUVA) compared with PUVA treatment alone in stage IB-IIA mycosis fungoides: final results from the EORTC Cutaneous Lymphoma Task Force phase III randomized clinical trial (NCT00056056). Br J Dermatol. 2012;167:678-87.

18. Fujimura T, Sato $Y$, Tanita $K$, et al. Case series of cutaneious $\mathrm{T}$-cell lymphomas treated with bexarotene-based therapy. J Dermatol. 2020;47:636-40.

19. Hamada T, Tokura Y, Sugaya M, et al. Long-term efficacy and safety of bexarotene for Japanese patients with cutaneous T-cell lymphoma: the results of a phase 2 study (B-1201). J Dermatol. 2019;46:557-63.

20. Hamada T, Sugaya M, Tokura Y, et al. Phase I/II study of the oral retinoid $X$ receptor agonist bexarotene in Japanese patients with cutaneous T-cell lymphomas. J Dermatol. 2017;44:135-42.

21. Duvic M, Hymes $K$, Heald $P$, et al. Bexarotene is effective and safe for treatment of refractory advanced-stage cutaneous T-cell lymphoma: multinational phase II-III trial results. J Clin Oncol. 2001;19:2456-71. 